\title{
Avaliação clínica da reconstrução do bíceps braquial com enxerto de tríceps*
}

\section{Clinical Evaluation of the Reconstruction of the Biceps Brachii using Triceps Graft}

\author{
Thiago Medeiros Storti ${ }^{10}$ Rafael Gomes Dias ${ }^{20}$ Gabriel Cesar Dias Dantas ${ }^{30}$ \\ Rafael Salomon Silva Faria2@ João Eduardo Simionatto ${ }^{20}$ Alexandre Firmino Paniago ${ }^{20}$
}

1 Instituto de Pesquisa e Ensino, Hospital Ortopédico e Medicina

Endereço para correspondência Rafael Salomon Silva Faria, Quadra Especializada, Brasília, DF, Brasil

2 Instituto do Ombro de Brasília, Brasília, DF, Brasil

${ }^{3}$ Centro Universitário de Brasília, Brasília, DF, Brasil 102 norte, praça Perdiz, Lote 05 , Condomínio Residencial Matisse Antares, Águas Claras, DF, 71.907-000, Brasil (e-mail: thiago_storti@hotmail.com).

Rev Bras Ortop 2021;56(5):656-663.

\section{Resumo \\ Palavras-chave \\ - autoenxerto \\ - cotovelo \\ - procedimentos ortopédicos \\ - lesões tendíneas \\ - membro superior \\ Objetivo Avaliação clínica e funcional do tratamento cirúrgico da lesão crônica do bíceps braquial distal, por aplicação de técnica cirúrgica com enxerto de tendão do tríceps braquial distal. \\ Métodos Estudo baseado em revisão de prontuários e avaliação clínica de pacientes com lesão crônica da inserção distal do bíceps braquial submetidos a tratamento cirúrgico entre fevereiro de 2015 e fevereiro de 2017. Durante o acompanhamento pós-operatório mínimo de 12 meses, 7 pacientes foram avaliados quanto à amplitude de movimento dos cotovelos operado e não operado, à força de flexão, à extensão e supinação dos membros superiores, determinadas com dinamômetro digital, ao teste do gancho, ao índice de satisfação, e às escalas Disabilities of the Arm, Shoulder and Hand (DASH) e Mayo Elbow Performance Score (MEPS). \\ Resultados À avaliação funcional pós-operatória, nenhum paciente relatou insatisfa- ção com o aspecto estético das incisões; todos ficaram satisfeitos/muito satisfeitos com a amplitude de movimento e a força do membro operado. Complicações neurovasculares, infecção de sítio cirúrgico, ou ruptura de tendão não foram observa- das. Nas escalas MEPS e DASH, todos os pacientes apresentaram pontuações iguais a 100 e 0 , respectivamente. A flexão média foi de $133,5^{\circ}$ no lado operado, contra $139,2^{\circ}$ no lado não operado. A extensão média foi de $5^{\circ}$ no lado operado, contra $0^{\circ}$ no lado não operado. A supinação foi de $86,5^{\circ}$ contra $90^{\circ}$, e a pronação foi de $80^{\circ}$ contra $80^{\circ}$, ao se comparar os lados operado e não operado, respectivamente. As forças médias de}

\footnotetext{
Estudo realizado no Hospital Ortopédico e Medicina Especializada (HOME), DF, Brasil.
}

recebido

12 de Fevereiro de 2020

aceito

28 de Outubro de 2020

Publicado on-line

Agosto 13, 2021
DOI https://doi.org/ $10.1055 / \mathrm{s}-0041-1729566$ ISSN 0102-3616.

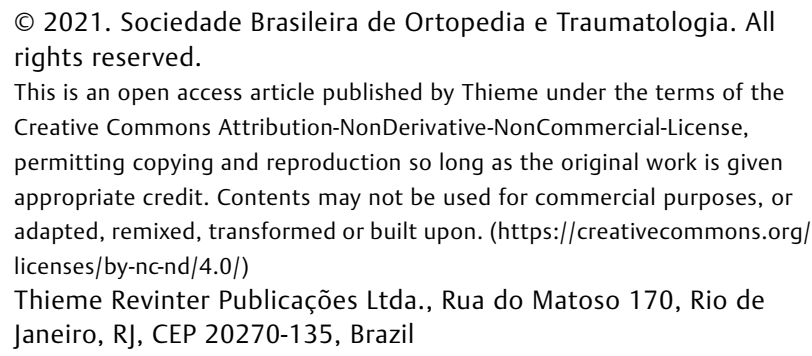

(c) 2021. Sociedade Brasileira de Ortopedia e Traumatologia. All rights reserved.

This is an open access article published by Thieme under the terms of the Creative Commons Attribution-NonDerivative-NonCommercial-License, permitting copying and reproduction so long as the original work is given appropriate credit. Contents may not be used for commercial purposes, or adapted, remixed, transformed or built upon. (https://creativecommons.org/ licenses/by-nc-nd/4.0/) Thieme Revinter Publicações Ltda., Rua do Matoso 170, Rio de Janeiro, RJ, CEP 20270-135, Brazil 


\begin{abstract}
Keywords

- autografting

- elbow

- orthopedic procedures

- tendon injuries

- upper extremity

Objective Clinical and functional evaluation of the surgical treatment for chronic injury of the distal biceps brachii applying a surgical technique with grafting of the distal triceps brachii tendon.

Methods A study based on a review of the medical records and clinical evaluation of the patients submitted to surgical treatment for chronic injury to the distal insertion of the biceps brachii between February 2015 and February 2017. In a 12-month-minimum postoperative follow-up, 7 patients were evaluated regarding the range of motion of the operated and non-operated elbows, flexion, upper-limb extension and supination with a digital dynamometer, the hook test, the satisfaction index, and the Disabilities of the Arm, Shoulder and Hand (DASH) and Mayo Elbow Performance Score (MEPS) intruments.

Results During the postoperative functional evaluation, no patient reported dissatisfaction with the esthetic outcome of the incisions, and all of them were satisfied/very satisfied with the range of motion and strength of the operated limb. No neurovascular complications, surgical site infection or tendon rupture were observed. On the MEPS and DASH scales, all patients scored 100 and 0 respectively. The mean flexion was of $133.5^{\circ}$ on the operated side, versus $139.2^{\circ}$ on the non-operated side. The mean extension was of $5^{\circ}$ on the operated side versus $0^{\circ}$ on the non-operated side. The supination was of $86.5^{\circ}$ versus $90^{\circ}$, and the pronation, $80^{\circ}$ versus $80^{\circ}$, when comparing the operated and non-operated sides respectively. The mean flexion, extension and supination corresponded respectively to $92.5 \%, 96.4 \%$ and $86.8 \%$ of those of the nonoperated limb.

Conclusion Recosntruction of the distal biceps brachii with triceps grafting seems to be an effective and safe option for the treatment of chronic distal biceps injuries.
\end{abstract}

flexão, extensão e supinação corresponderam, respectivamente, a 92,5\%, 96,4\%, e $86,8 \%$ das forças do membro não operado.

Conclusão A reconstrução do bíceps braquial distal com enxerto de tríceps parece ser uma opção eficaz e segura para o tratamento de lesões crônicas do bíceps distal.

\section{Introdução}

O bíceps braquial é o supinador primário e flexor secundário do antebraço. ${ }^{1}$ Rupturas do tendão distal do bíceps braquial são lesões raras, e geralmente afetam o membro dominante de homens de meia-idade. A lesão é normalmente caracterizada por contração excêntrica com o cotovelo em $90^{\circ}$ de flexão. ${ }^{2} \mathrm{O}$ quadro clínico é caracterizado por dor aguda, edema, e equimoses locais, associados a um estalido audível durante a lesão, presença de uma fenda proximal à fossa cubital, e perda da força de supinação do antebraço e de flexão do cotovelo.

A perda significativa da força de flexão, e, mais pronunciada, da força de supinação são bastante associadas a rupturas crônicas. ${ }^{2}$ Os principais fatores de risco são uso de anabolizantes, levantamento de peso, e tabagismo. ${ }^{3} \mathrm{~A}$ tendinopatia degenerativa e algumas doenças endócrinas também são implicadas nas rupturas do tendão distal do bíceps braquial. ${ }^{4} \mathrm{~A}$ ruptura é considerada crônica após 4 a 6 semanas de lesão. ${ }^{1}$ Nestes casos, a unidade musculotendínea se retrai, e há formação de fibrose, o que dificulta o reparo da tuberosidade radial. ${ }^{5-7}$ Seu tratamento conservador tem gerado resultados insatisfatórios. ${ }^{8}$
Diversos procedimentos foram descritos para o tratamento de rupturas crônicas do tendão distal do bíceps braquial, como tenodese braquial e o uso de enxertos de tendão, como palmar longo, calcâneo, tensor da fáscia lata e semitendinoso. ${ }^{4,9,10} \mathrm{O}$ objetivo deste estudo foi apresentar os resultados clínicos e funcionais da reconstrução distal do bíceps braquial com enxerto de tríceps distal central, com fixação de duas âncoras bioabsorvíveis na tuberosidade radial de pacientes com lesão do bíceps distal por mais de 4 semanas. Esta técnica foi recentemente publicada pelos autores.

\section{Material e Métodos}

De fevereiro de 2015 a fevereiro de 2017, 7 pacientes com lesão do bíceps distal havia mais de 28 dias foram submetidos à reconstrução do bíceps distal com enxerto de tríceps (-Tabela 1). Todos os pacientes eram do sexo masculino, e sua idade média era de 45 anos (gama: 30 a 60 anos). A lesão foi no membro dominante em três casos, e três pacientes relataram o uso de esteroides anabolizantes. 


\begin{tabular}{|c|c|c|c|c|c|c|c|c|c|c|c|c|c|c|c|}
\hline 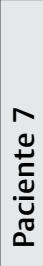 & 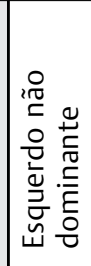 & 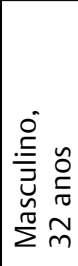 & 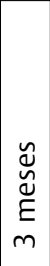 & $E$ & 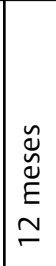 & 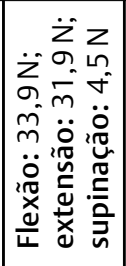 & 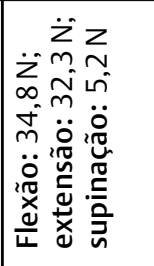 & 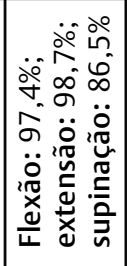 & 官 & $\therefore$ & ৪০ & $\stackrel{\infty}{\infty}$ & 음 & 0 & 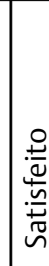 \\
\hline 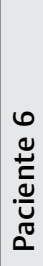 & 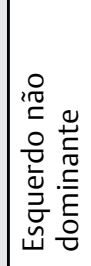 & 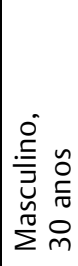 & 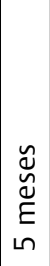 & $E$ & 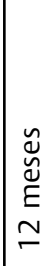 & 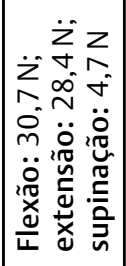 & 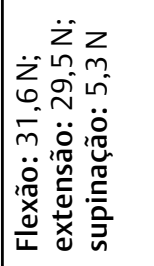 & 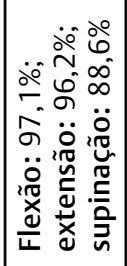 & 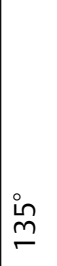 & $\therefore$ & 迆 & $\stackrel{\infty}{\infty}$ & 음 & 0 & 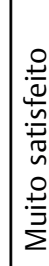 \\
\hline 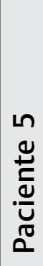 & 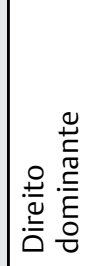 & 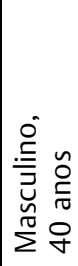 & 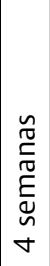 & E & 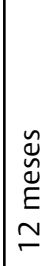 & 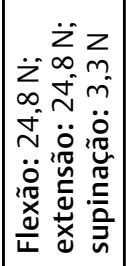 & 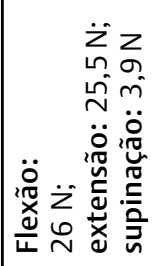 & 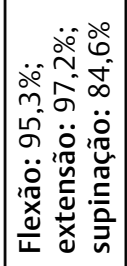 & $\stackrel{\stackrel{n}{m}}{r}$ & $\therefore$ & б & $\infty$ & $=$ & 0 & $\begin{array}{l}\tilde{T}_{0}^{-} \\
\tilde{n}\end{array}$ \\
\hline
\end{tabular}

\begin{tabular}{|c|c|c|c|c|c|c|c|c|c|c|c|c|c|c|c|}
\hline & 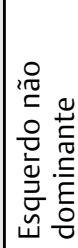 & 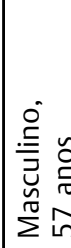 & 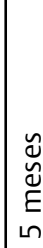 & $\frac{i \pi}{Z}$ & 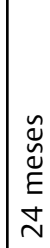 & 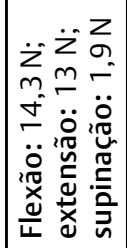 & 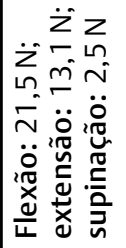 & 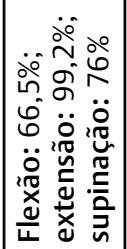 & 守 & $\stackrel{\circ}{-}$ & ஜ̊ & $i_{\infty}^{\circ}$ & $\stackrel{0}{-}$ & 0 & 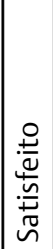 \\
\hline
\end{tabular}

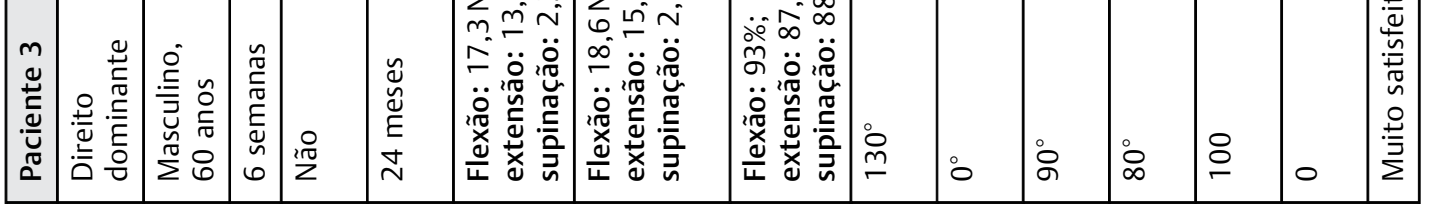

\begin{tabular}{|c|c|c|c|c|c|c|c|c|c|c|c|c|c|c|c|}
\hline 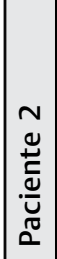 & 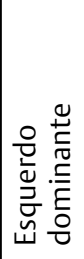 & 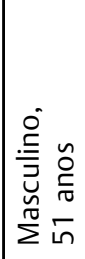 & 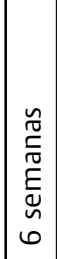 & $\begin{array}{l}\frac{0}{2 \pi} \\
\frac{2 \pi}{2}\end{array}$ & \begin{tabular}{|l}
$\breve{y}$ \\
$\tilde{ٌ}$ \\
$\tilde{E}$ \\
$\emptyset$ \\
$\tilde{m}$
\end{tabular} & 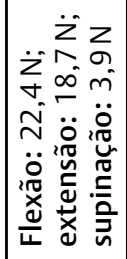 & 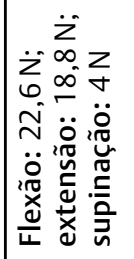 & 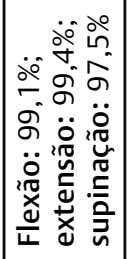 & ì & $\therefore$ & $\stackrel{\infty}{\infty}$ & $\stackrel{\infty}{\infty}$ & 잉 & 10 & 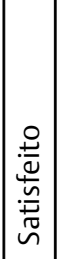 \\
\hline 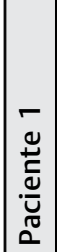 & 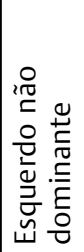 & 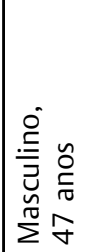 & \begin{tabular}{|l}
$\breve{ٌ}$ \\
$\breve{ٌ}$ \\
$\tilde{\Xi}$ \\
$m$
\end{tabular} & 竞 & \begin{tabular}{|l}
$\breve{ٌ}$ \\
$\breve{ٌ ~}$ \\
$\underline{\Xi}$ \\
$\check{\infty}$
\end{tabular} & 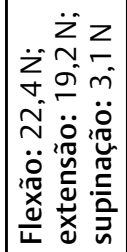 & 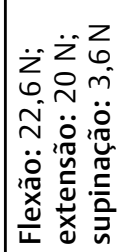 & 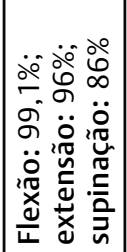 & 吕 & in & 战 & $\infty$ & ○ & 10 & 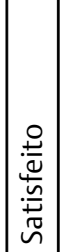 \\
\hline & 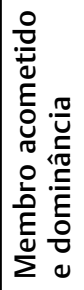 & 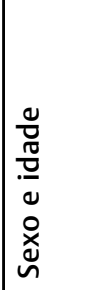 & 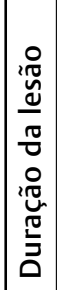 & 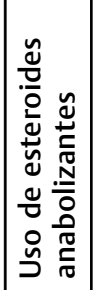 & 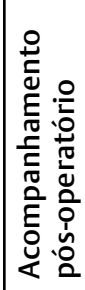 & 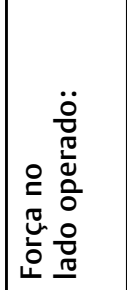 & 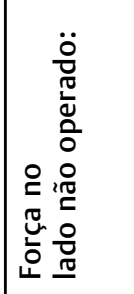 & 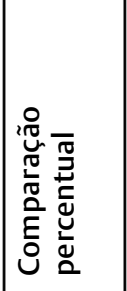 & 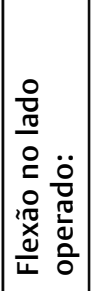 & 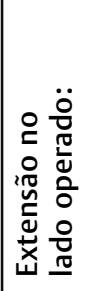 & 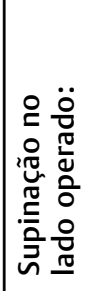 & 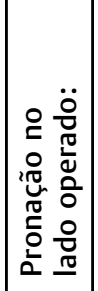 & 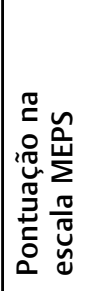 & 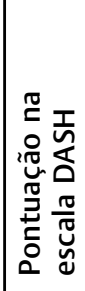 & 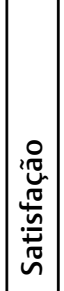 \\
\hline
\end{tabular}


O período médio de acompanhamento pós-operatório foi de 18 meses (gama: 12 a 36 meses). 0 principal mecanismo de lesão foi a contração excêntrica do bíceps braquial. Os pacientes foram submetidos à cirurgia em média 3 meses após a lesão (gama: 1 a 5 meses). Ao exame físico, apresentavam perda da força de flexão do cotovelo, principalmente da supinação. 0 teste do gancho foi positivo para lesão em todos os pacientes no pré-operatório, e negativo na última avaliação do acompanhamento pós-operatório. Todos tiveram o diagnóstico confirmado por ressonância magnética realizada para avaliação do grau de lesão e encurtamento tendíneo.

As complicações e os riscos do tratamento foram explicados aos pacientes, bem como a necessidade de enxerto de tecido autólogo em caso de impossibilidade de reinserção primária do tendão bicipital. No período pós-operatório, as escalas Disability of Arm Shoulder and Hand (DASH) e Mayo Elbow Performance Score (MEPS) foram aplicadas, e o teste de movimentação dos cotovelos operado e não operado foi realizado com goniômetro manual. Além disso, as forças de flexão, extensão e supinação dos membros operado e não operado foram avaliadas e comparadas. Um dinamômetro digital (Lafayette Hand-Held Dynamoneter, modelo 01163, Lafayette Instrument, Lafayette, IN, EUA) foi usado para medir as forças de flexão, extensão e supinação; um cabo de madeira devidamente marcado foi empregado para facilitar a análise da supinação sem interferir no momento das forças aplicadas. Quatro medições foram feitas, sempre pelo mesmo avaliador, e a média das três últimas foi obtida. A primeira medida foi desconsiderada para evitar o viés de aprendizado da forma de medida pelo paciente. Por fim, os pacientes foram avaliados quanto ao grau de satisfação (insatisfeito, não satisfeito, satisfeito, e muito satisfeito).

O estudo foi aprovado pelo comitê de ética da instituição sob o número CAAE 69377517.5.0000.0023.

\section{Técnica Cirúrgica}

As cirurgias foram realizadas sob anestesia geral com bloqueio locorregional do plexo braquial, em decúbito dorsal horizontal e sem torniquetes. Optamos pela técnica de duas incisões descrita por Boyd e Anderson, ${ }^{11}$ e modificada por Morrey et al., ${ }^{7}$ com utilização de enxerto do tendão distal do tríceps braquial. O critério para definir a reconstrução do tendão foi a impossibilidade de excursão do tendão remanescente até a tuberosidade radial, mesmo após a liberação da aponeurose do músculo bíceps braquial (lacertus fibrosus). Uma incisão transversal de aproximadamente $3 \mathrm{~cm}$ foi feita na fossa cubital anterior. O tendão do bíceps foi facilmente capturado, com tensão da pele em sentido proximal, e removido dos tecidos profundos. A porção mais distal do tendão degenerado foi ressecada e reparada com sutura de Bunnell com fio não absorvível $n^{\circ} 5$ (-Fig. 1). Em seguida, a tuberosidade radial foi palpada, e uma pinça Kelly curva foi passada pelo túnel do tendão do bíceps entre a ulna e o rádio, avançando até que seu ápice fosse palpado na face dorsal do antebraço proximal. A segunda incisão foi feita na pinça. A tuberosidade foi exposta por meio de divulsão muscular com o antebraço em pronação máxima. A tuberosidade radial foi

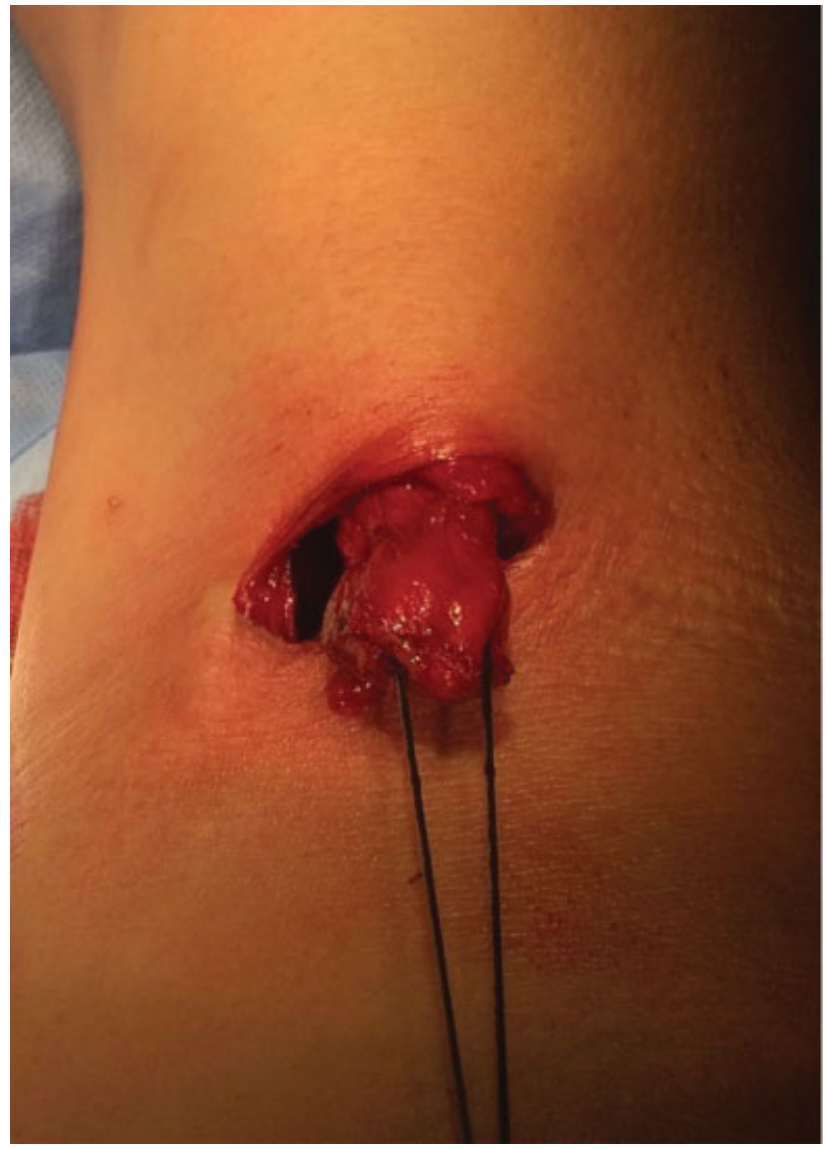

Fig. 1 Tendão do bíceps distal após a liberação mostrando impossibilidade de reparo direto.

escarificada até começar a sangrar, e nela foram posicionadas duas âncoras bioabsorvíveis de 2,9 mm e carga dupla.

Depois, o enxerto do tríceps braquial foi coletado sem fragmentos ósseos do olécrano, por meio de incisão longitudinal posterior e dissecção subcutânea até a exposição de seu tendão. Uma faixa de $1 \mathrm{~cm}$ de largura e $10 \mathrm{~cm}$ de comprimento de sua porção média foi retirada, sem a necessidade de exploração do nervo ulnar. Posteriormente, as bordas medial e lateral da porção removida foram abordadas, e a lacuna deixada foi fechada (-Fig. 2 ).

A extremidade mais distal do enxerto foi fixada na tuberosidade por meio de quatro suturas ancoradas em U (-Fig. 3). A outra extremidade do tendão foi passada para a região da incisão da fossa antecubital por meio de suturas de Krackow com fio não absorvível $n^{\circ} 5$, para tração do tendão pelo túnel anteriormente ocupado pelo tendão do bíceps. 0 bíceps foi mobilizado e tracionado com a pinça de Allis. O cotovelo foi posicionado entre $40^{\circ}$ e $60^{\circ}$ de flexão, com o antebraço em supinação total. $O$ enxerto foi submetido a tração moderada e o coto do tendão recebeu tração distal. As duas estruturas foram inicialmente estabilizadas com suturas em $U$ de fio não absorvível $n^{\circ} 5$, e, em seguida, vários pontos simples separados foram aplicados em suas bordas (-Fig. 4). Concluída a reconstrução, as feridas foram fechadas. Curativos compressivos foram colocados, e a imobilização foi feita com tala braquial gessada, mantendo o cotovelo em flexão de $90^{\circ}$ e o antebraço em supinação branda. 

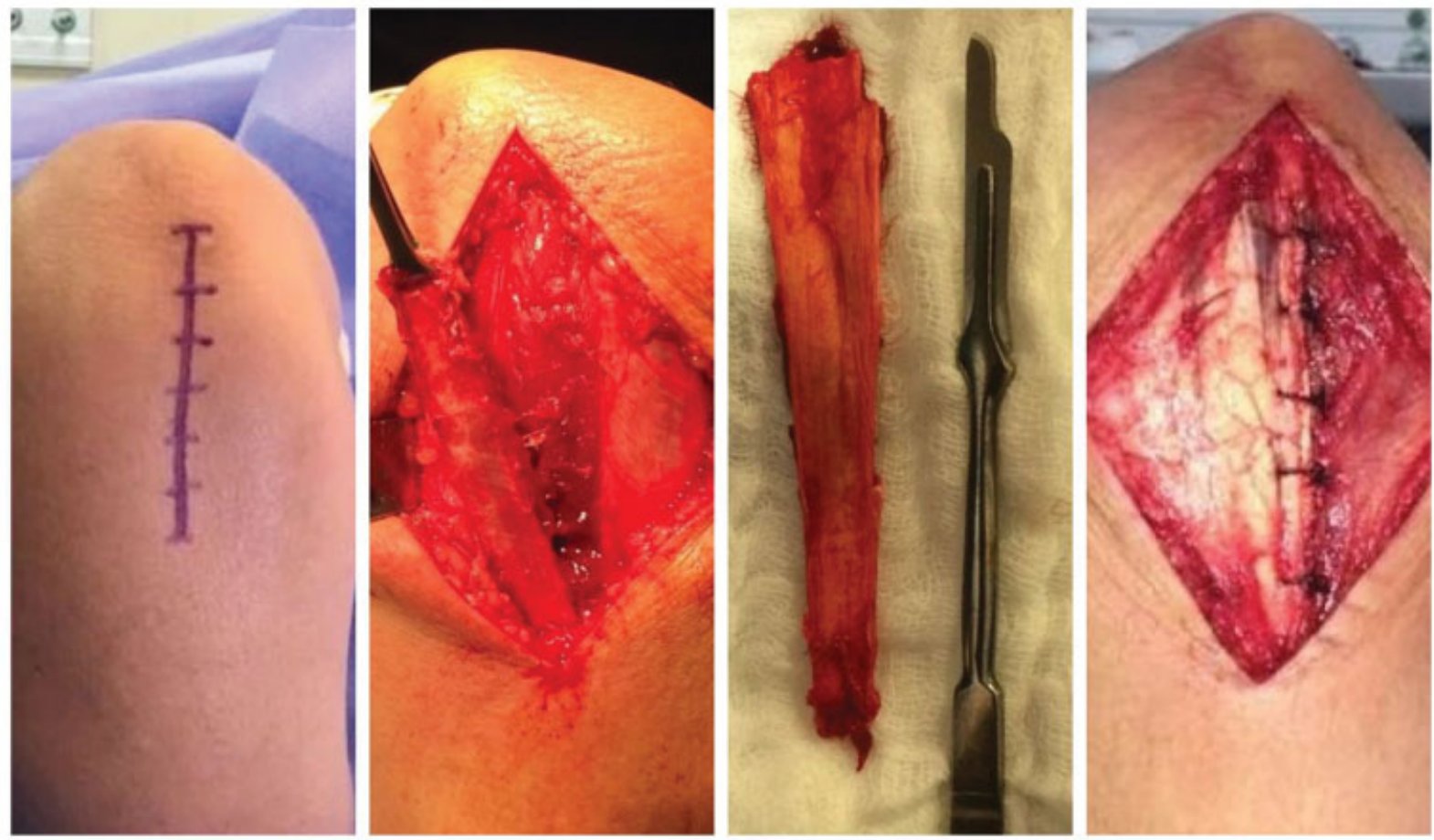

Fig. 2 I) Incisão para obtenção do enxerto de tendão do tríceps; II) ressecção da parte central do tendão com $10 \mathrm{~cm} \times 1 \mathrm{~cm}$; III) enxerto de tendão do tríceps; IV) sutura do tendão do tríceps remanescente.

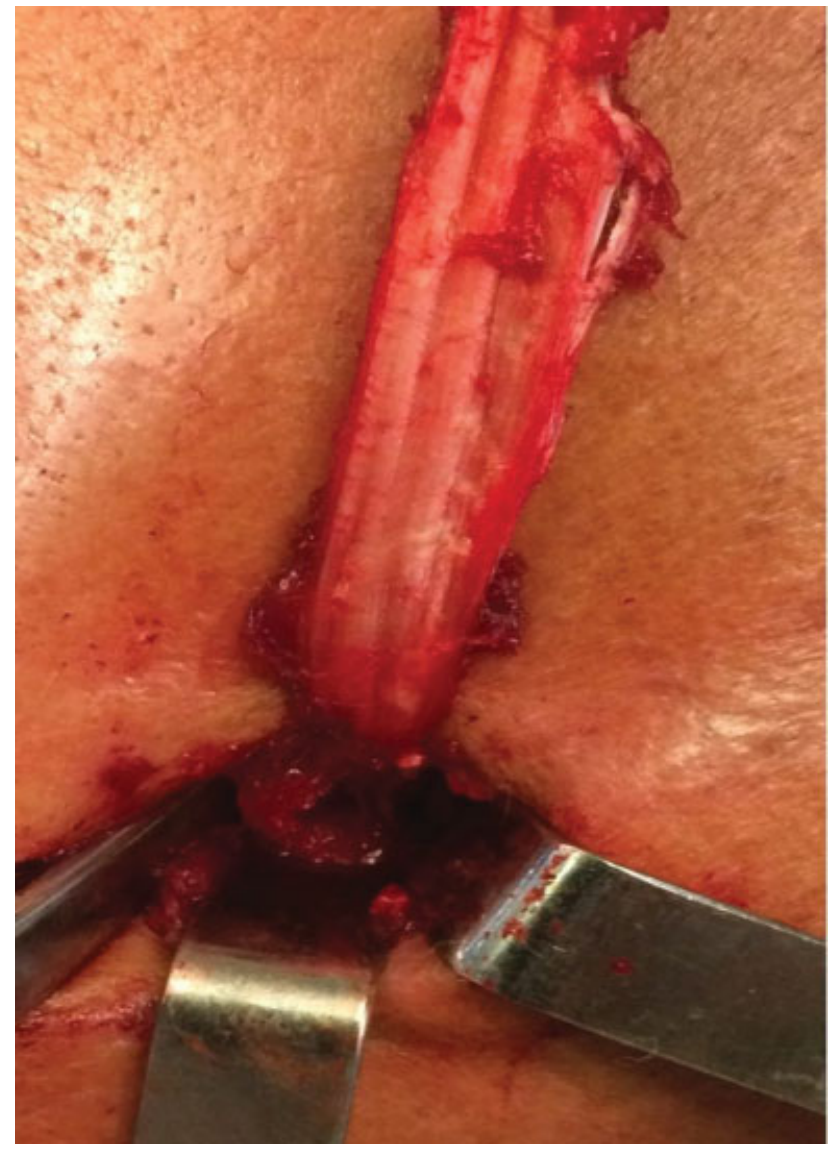

Fig. 3 A extremidade distal do enxerto ligada à tuberosidade do bíceps.

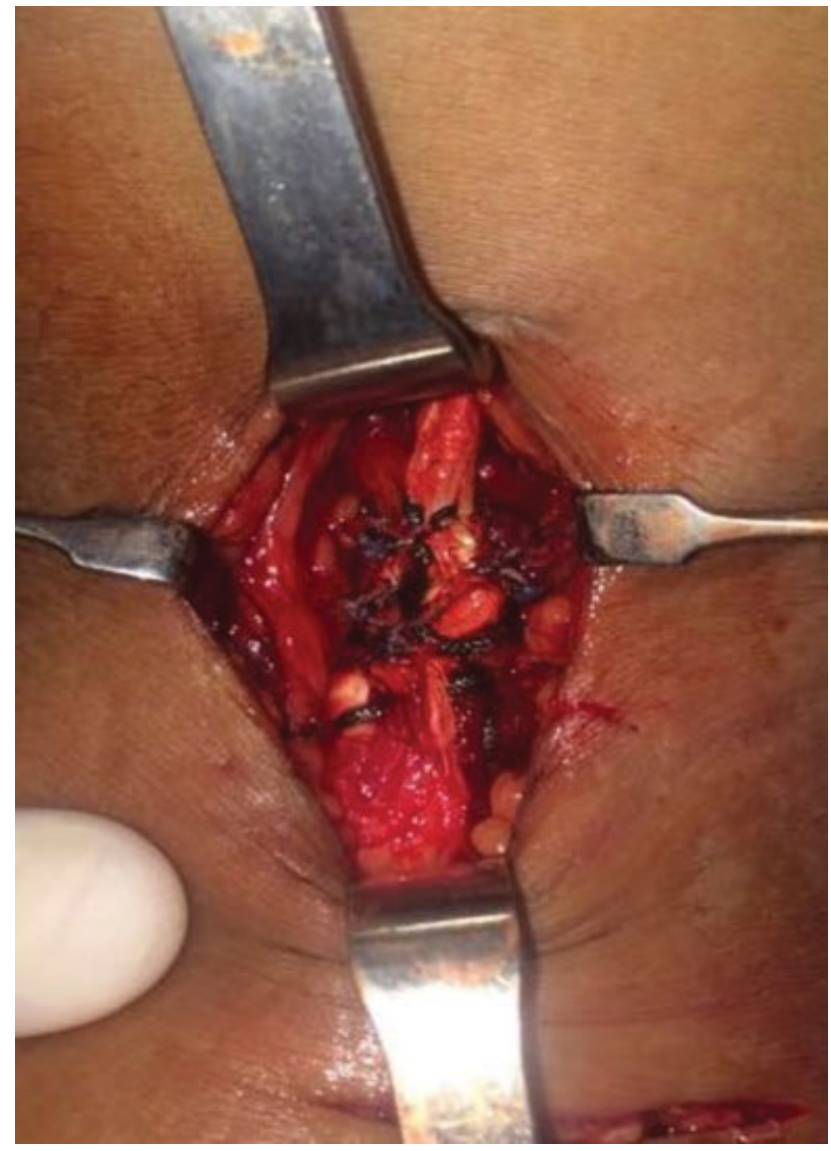

Fig. 4 Sutura entre a parte proximal do enxerto de tríceps e o tendão distal do bíceps. 
A imobilização foi mantida por duas semanas, quando começou o tratamento fisioterapêutico. A princípio, exercícios de flexão passiva e extensão ativa limitada foram realizados com o antebraço supinado, além de pronaçãosupinação passiva e ativa de até $50^{\circ}$. O paciente manteve o membro na tipoia enquanto não estava em fisioterapia. Essa fase continuou até o final das quatro semanas, quando o ganho de flexão e supinação ativa começou sem carga, e o paciente foi aconselhado a ficar sem a tipoia. Exercícios leves de fortalecimento muscular começaram a partir da sexta semana, com aumento progressivo da carga.

\section{Análise Estatística}

A análise descritiva apresentou os dados observados em tabelas, expressos por medidas de tendência central e dispersão adequada.

A análise inferencial foi composta pelo teste de MannWhitney para verificar a presença de diferença significativa no parâmetro de força entre os lados operado e não operado.

A normalidade na distribuição dos dados numéricos foi avaliada pelo teste de Shapiro-Wilk e pela análise gráfica dos histogramas. O nível de $5 \%$ foi adotado como critério de determinação de significância. A análise estatística foi realizada no programa Statistical Package for the Social Sciences (SPSS, IBM Corp., Armonk, NY, EUA), versão 26.

\section{Resultados}

Todos os pacientes ficaram satisfeitos/muito satisfeitos com os resultados funcionais. A flexão média foi de aproximadamente $133,5^{\circ}$, correspondendo a $95,9 \%$ do valor observado no membro não operado. A extensão média foi de $3,5^{\circ}$, e correspondeu a $97,5 \%$ da do membro não operado. Um paciente apresentou contratura em flexão de $10^{\circ}$ mantida na última consulta de acompanhamento (24 meses após a cirurgia). A supinação média foi de $86,5^{\circ}$, e o nível de pronação foi de $80^{\circ}$, o que correspondeu a $96 \%$ e $100 \%$ dos valores observados no membro contralateral, respectivamente (-Tabela 2 ).

Todos os pacientes apresentaram excelentes resultados na escala MEPS, com 100 pontos. No questionário DASH, todos os pacientes apresentaram resultado 0 . A força de flexão média foi de $23,7 \mathrm{~N}$, enquanto a de supinação foi de $3,4 \mathrm{~N}$, e a de extensão, 21,3 N; esses valores corresponderam, respectivamente, a $92,5 \%, 86,8 \%$ e $96,4 \%$ da média da força do lado não operado (-Tabela $\mathbf{3}$ ).

A análise dos dados por meio de abordagem não paramétrica foi proposta devido ao diminuto tamanho da amostra. Além disso, alguns parâmetros em estudo não apresentaram distribuição normal (Gaussiana) segundo o teste de ShapiroWilk. Portanto, as medidas mais adequadas para resumir esses dados foram quartis (mediana e intervalo interquartil: Q1-Q3). Não houve diferença estatisticamente significativa no parâmetro de força.

Complicações neurovasculares, infecção de sítio cirúrgico, nova ruptura de tendão, fratura cortical do rádio ou ossificação heterotópica não foram observadas.
Tabela 2 Comparação da amplitude de movimento entre os lados operado e não operado

\begin{tabular}{|c|c|c|}
\hline Flexão & $\begin{array}{l}\text { Média, valor mínimo } \\
\text { e valor máximo }\end{array}$ & \\
\hline Lado operado & $133,5^{\circ}, 130^{\circ}, 140^{\circ}$ & \multirow[t]{2}{*}{$95,9 \%$} \\
\hline Lado não operado & $139,2^{\circ}, 135^{\circ}, 140^{\circ}$ & \\
\hline \multicolumn{3}{|l|}{ Extensão } \\
\hline Lado operado & $3,5^{\circ}, 10^{\circ}, 0^{\circ}$ & \multirow[t]{2}{*}{$97,5 \%$} \\
\hline Lado não operado & $0^{\circ}, 0^{\circ}, 0^{\circ}$ & \\
\hline \multicolumn{3}{|l|}{ Supinação } \\
\hline Lado operado & $86,5^{\circ}, 80^{\circ}, 90^{\circ}$ & \multirow[t]{2}{*}{$96 \%$} \\
\hline Lado não operado & $90^{\circ}, 90^{\circ}, 95^{\circ}$ & \\
\hline \multicolumn{3}{|l|}{ Pronação } \\
\hline Lado operado & $80^{\circ}, 80^{\circ}, 80^{\circ}$ & \multirow[t]{2}{*}{$100 \%$} \\
\hline Lado não operado & $80^{\circ}, 80^{\circ}, 80^{\circ}$ & \\
\hline
\end{tabular}

Tabela 3 Comparação de força entre os lados operado e não operado

\begin{tabular}{|c|c|c|}
\hline Flexão & $\begin{array}{l}\text { Média, mediana, } \\
\text { intervalo interquartil }\end{array}$ & $p$ \\
\hline Lado operado & $23,7 \mathrm{~N}, 22,4 \mathrm{~N}, 17,3-30,7$ & \multirow[t]{3}{*}{0,48} \\
\hline Lado não operado & $25,4 \mathrm{~N}, 22,6 \mathrm{~N}, 21,5-31,6$ & \\
\hline$\%$ & $92,5,97,2,93,0-99,1$ & \\
\hline \multicolumn{3}{|l|}{ Supinação } \\
\hline Lado operado & $3,4 \mathrm{~N}, 3,3 \mathrm{~N}, 2,2-4,5$ & \multirow[t]{3}{*}{0,37} \\
\hline Lado não operado & $3,9 \mathrm{~N}, 3,9 \mathrm{~N}, 2,5-5,2$ & \\
\hline$\%$ & $86,8,86,5,84,6-88,7$ & \\
\hline \multicolumn{3}{|l|}{ Extensão } \\
\hline Lado operado & $21,3 \mathrm{~N}, 19,2 \mathrm{~N}, 13,4-28,4$ & \multirow[t]{3}{*}{0,65} \\
\hline Lado não operado & $22,1 \mathrm{~N}, 20,0 \mathrm{~N}, 15,3-29,5$ & \\
\hline$\%$ & $96,4,97,3,96,0-99,2$ & \\
\hline
\end{tabular}

\section{Discussão}

O reparo primário de uma ruptura crônica do bíceps braquial distal é tecnicamente desafiador. A tenodese não anatômica do músculo braquial tem sido proposta como opção terapêutica. No entanto, apesar do alto índice de satisfação dos pacientes submetidos a este procedimento, Klonz et al. ${ }^{12}$ observaram que metade deles perdeu mais de $50 \%$ da força de supinação. 0 risco de fraqueza em supinação após a utilização desta técnica pode ser inaceitável em pacientes com alta demanda funcional. Diversas técnicas de reconstrução do bíceps braquial distal foram descritas, e diferem em termos de acesso, escolha de enxerto e tipo de fixação. ${ }^{1,2,5,6}$ Tanto autoenxertos quanto aloenxertos têm sido usados com esta finalidade.

Várias opções de aloenxerto foram descritas na literatura, 1,13,14 inclusive de tendão calcâneo, semitendinoso, tibial anterior e grácil. Em relação a autoenxertos, , 2,5,6 há descrições do uso da fáscia lata, do semitendinoso, e do 
palmar longo. Não encontramos na literatura uma descrição do uso do tendão distal do tríceps braquial com esse fim. Planejamos a utilização desse tendão como autoenxerto em rupturas crônicas do bíceps braquial distal para evitar os inconvenientes do período de recuperação observados quando a área doadora não está localizada na mesma articulação do que a área receptora. Além disso, outras vantagens são a presença desse tendão doador em toda a população, a ausência de riscos neurovasculares durante sua coleta, e a possibilidade de comprimentos e tamanhos variáveis conforme a necessidade.

Martin et al. ${ }^{15}$ avaliaram as características biomecânicas do enxerto da porção central do tríceps braquial, e concluíram que o enxerto de tríceps é comparável ao tendão palmar longo quanto à carga final de falha e rigidez. Além disso, observaram que o tendão do tríceps apresenta maior deformação, mas sem significância estatística. Em outro estudo biomecânico, Baumfeld et al. ${ }^{16}$ avaliaram as propriedades do tríceps distal medial, central e lateral, e concluíram que a porção lateral é significativamente mais delgada e menos rígida do que as porções central e medial, e que a porção central do tríceps braquial apresenta uma carga final de falha de $704 \mathrm{~N}$, contra $357 \mathrm{~N}$ da do tendão palmar longo.

Wiley et al. ${ }^{2}$ compararam dois grupos de pacientes com rupturas crônicas do bíceps braquial distal, um submetido ao tratamento conservador, e outro, à reconstrução com autoenxerto de semitendinoso pela técnica de duas incisões. Os autores concluíram que os pacientes submetidos à reconstrução obtiveram melhora da força de flexão e supinação em comparação àqueles tratados de forma conservadora.

Hallam e Bain ${ }^{10}$ avaliaram nove pacientes submetidos ao reparo com enxerto autólogo de semitendinoso, fixação com placa de Endobutton (Smith \& Nephew, Inc., Andover, MA, EUA), e abordagem anterior em S. Como no presente estudo, também observaram excelente pontuação na escala MEPS em todos os casos, amplitude de movimento próxima ao norma,l e ausência de complicações pós-operatórias.

Terra et al. ${ }^{17}$ avaliaram 8 pacientes após o reparo direto de lesões crônicas, com tempo médio entre a lesão e a cirurgia de 71,8 dias (gama: 28 a 180 dias). Utilizaram a abordagem anterior, e, como método de fixação, Endobutton associado a parafuso de interferência. Esses autores também obtiveram resultados excelentes na escala MEPS, mas observaram força de flexão de $79,25 \%$ e de supinação de $89,75 \%$ em relação aos valores contralaterais. No presente estudo, a força de supinação foi semelhante, mas a força de flexão foi superior. Além disso, em nossa série, o reparo direto das lesões não foi possível, nem mesmo com a flexão do cotovelo.

Usando aloenxerto de tendão calcâneo, Sanchez-Sotelo et al., ${ }^{13}$ em seu estudo com 4 pacientes, apresentaram excelentes resultados na MEPS (100 pontos em todos os casos), com amplitude de movimento e força normais em relação ao lado contralateral em 2 pacientes, e um pouco menores nos outros 2 .

Há diversas opções de fixação do tendão na tuberosidade radial (túnel ósseo, parafuso de interferência, Endobutton, e suturas ancoradas). O Endobutton tem a maior resistência biomecânica, seguido pelas suturas ancoradas. Porém, não há diferença estatisticamente significativa entre o Endobutton e suturas ancoradas submetidos a forças fisiológicas. ${ }^{18,19}$ Além disso, existe a possibilidade de reparo do tendão com Endobutton e parafuso de interferência nas lesões crônicas do bíceps distal, o que permite uma fixação mais rígida e resistente, com dois implantes e reabilitação mais rápida. ${ }^{17}$ No entanto, as técnicas de ancoragem têm resultados clínicos e funcionais ideais. ${ }^{20}$

Embora ainda se debata o melhor acesso para fixação das rupturas do tendão distal do bíceps, seja por incisão dupla ou única, estudos recentes ${ }^{21,22}$ mostram uma diferença insignificante de resultados e complicações entre as duas técnicas. A escolha do melhor acesso para essas patologias deve ser orientada pela experiência e familiaridade do cirurgião.

Os pontos negativos do presente estudo são a ausência de dados para comparação entre o pré e pós-operatório devido ao seu caráter retrospectivo, a amostra limitada de pacientes $(\mathrm{n}=7)$, problema também observado na maioria dos estudos da literatura sobre o assunto, e o curto período de acompanhamento dos pacientes.

\section{Conclusão}

O enxerto da faixa central do tendão do tríceps apresenta características biomecânicas adequadas para a reconstrução do bíceps distal. Além disso, tem como vantagens a segurança de sua coleta e a possibilidade de retirada de enxertos de tamanhos variáveis. Observamos que, por se tratar de uma lesão rara, há muita dificuldade em se fazer grandes estudos prospectivos para comparação dos métodos de tratamento cirúrgico dessa lesão. Porém, a reconstrução distal do bíceps braquial com enxerto de tríceps por dupla incisão, com fixação da tuberosidade radial com duas âncoras de sutura bioabsorvíveis, parece ser uma opção eficaz e segura para o tratamento das lesões crônicas do bíceps distal, com bons resultados clínicos e funcionais.

\section{Suporte Financeiro}

Este trabalho teve suporte do Instituto de Pesquisa e Ensino Home, Brasília, DF, Brasil.

Conflito de Interesses

Os autores declaram não haver conflito de interesses.

\section{Referências}

1 Darlis NA, Sotereanos DG. Distal biceps tendon reconstruction in chronic ruptures. J Shoulder Elbow Surg 2006;15(05): 614-619

2 Wiley WB, Noble JS, Dulaney TD, Bell RH, Noble DD. Late reconstruction of chronic distal biceps tendon ruptures with a semitendinosus autograft technique. J Shoulder Elbow Surg 2006;15 (04):440-444

3 Safran MR, Graham SM. Distal biceps tendon ruptures: incidence, demographics, and the effect of smoking. Clin Orthop Relat Res 2002;(404):275-283

4 Geaney LE, Mazzocca AD. Biceps brachii tendon ruptures: a review of diagnosis and treatment of proximal and distal biceps tendon ruptures. Phys Sportsmed 2010;38(02):117-125 
5 Levy HJ, Mashoof AA, Morgan D. Repair of chronic ruptures of the distal biceps tendon using flexor carpi radialis tendon graft. Am J Sports Med 2000;28(04):538-540

6 Hang DW, Bach BR Jr, Bojchuk J. Repair of chronic distal biceps brachii tendon rupture using free autogenous semitendinosus tendon. Clin Orthop Relat Res 1996;(323):188-191

7 Morrey BF, Askew LJ, An KN, Dobyns JH. Rupture of the distal tendon of the biceps brachii. A biomechanical study. J Bone Joint Surg Am 1985;67(03):418-421

8 Flint JH, Wade AM, Giuliani J, Rue JP. Defining the terms acute and chronic in orthopaedic sports injuries: a systematic review. Am J Sports Med 2014;42(01):235-241

9 Bell RH, Wiley WB, Noble JS, Kuczynski DJ. Repair of distal biceps brachii tendon ruptures. J Shoulder Elbow Surg 2000;9(03): 223-226

10 Hallam P, Bain GI. Repair of chronic distal biceps tendon ruptures using autologous hamstring graft and the Endobutton. J Shoulder Elbow Surg 2004;13(06):648-651

11 Boyd HB, Anderson MD. A method for reinsertion of the distal biceps brachii tendon. J Bone Joint Surg Am 1961;43(07):1041-1043

12 Klonz A, Loitz D, Wöhler P, Reilmann H. Rupture of the distal biceps brachii tendon: isokinetic power analysis and complications after anatomic reinsertion compared with fixation to the brachialis muscle. J Shoulder Elbow Surg 2003;12(06):607-611

13 Sanchez-Sotelo J, Morrey BF, Adams RA, O'Driscoll SW. Reconstruction of chronic ruptures of the distal biceps tendon with use of an achilles tendon allograft. J Bone Joint Surg Am 2002;84(06): 999-1005

14 Patterson RW, Sharma J, Lawton JN, Evans PJ. Distal biceps tendon reconstruction with tendoachilles allograft: a modification of the endobutton technique utilizing an ACL reconstruction system. J Hand Surg Am 2009;34(03):545-552

15 Martin CR, Hildebrand KA, Baergen J, Bitting S. Triceps tendon fascia for collateral ligament reconstruction about the elbow: a clinical and biomechanical evaluation. Am J Orthop 2011;40(09): E163-E169

16 Baumfeld JA, van Riet RP, Zobitz ME, Eygendaal D, An KN, Steinmann SP. Triceps tendon properties and its potential as an autograft. J Shoulder Elbow Surg 2010;19(05):697-699

17 Terra BB, Rodrigues LM, Lima AL, Cabral BC, Cavatte JM, De Nadai A. Direct repair of chronic distal biceps tendon tears. Rev Bras Ortop 2016;51(03):303-312

18 Mazzocca AD, Burton KJ, Romeo AA, Santangelo S, Adams DA, Arciero RA. Biomechanical evaluation of 4 techniques of distal biceps brachii tendon repair. Am J Sports Med 2007;35(02): 252-258

19 Olsen JR, Shields E, Williams RB, Miller R, Maloney M, Voloshin I. A comparison of cortical button with interference screw versus suture anchor techniques for distal biceps brachii tendon repairs. J Shoulder Elbow Surg 2014;23(11):1607-1611

20 Sarda P, Qaddori A, Nauschutz F, Boulton L, Nanda R, Bayliss N. Distal biceps tendon rupture: current concepts. Injury 2013;44 (04):417-420

21 Grewal R, Athwal GS, MacDermid JC, et al. Single versus doubleincision technique for the repair of acute distal biceps tendon ruptures: a randomized clinical trial. J Bone Joint Surg Am 2012; 94(13):1166-1174

22 Keener JD. Controversies in the surgical treatment of distal biceps tendon ruptures: single versus double-incision repairs. J Shoulder Elbow Surg 2011;20(2, Suppl)S113-S125 